\title{
A HILBERT INTEGRAL INEQUALITY WITH HURWITZ ZETA FUNCTION
}

\author{
He LePing And GaO MingZhe
}

Abstract. It is shown that an extension of the Hilbert integral inequality can be established by introducing two parameters and a proper logarithm function. The constant factor expressed by product of the gamma function and the Hurwitz Zeta function is proved to be the best possible. And base on it, some interesting special results are enumerated. As applications, some equivalent forms are given.

Mathematics subject classification (2010): 26D15, 33E20.

Keywords and phrases: Hilbert integral inequality, gamma function, Hurwitz Zeta function, Bernoulli number, the best constant.

\section{REFERENCES}

[1] G. H. Hardy, J. E. Littlewood, G. Polya, Inequalities, Cambridge Univ. Press, Cambridge, 1952.

[2] Kuang Jichang, Applied Inequalities, 3nd. Ed, Shandong Science and Technology Press, Jinan, 2004.

[3] Yang Bicheng, On a Generalization of a Hilbert's Type Integral Inequality and Its Applications, Math. Appl., Vol 16, 2 (2003), 82-86.

[4] Zhou Yu, Gao Xuemei and Gao Mingzhe, An Extension of the Hilbert's Integral Inequality, Journal of Inequalities and Applications, Volume 2009, Article ID 158690, 12 pages, 2009.

[5] Gao Mingzhe, Hsu Lizhi, A Survey of Various Refinements and Generalizations of Hilbert's Inequalities, J. Math. Res. \& Exp., Vol. 25, 2 (2005), 227-243.

[6] Yang Bicheng, On a Basic Hilbert-type Integral Inequality and Extensions, College Mathematics, Vol. 24, 1 (2008), 87-92.

[7] Hong Yong, All-sided Generalization about Hardy-Hilbert's Integral Inequalities, Acta Mathematica Sinica, Vol. 44, 4 (2001), 619-626.

[8] Hu Ke, On Hilbert's Inequality, Chin. Ann. Math., Ser. B, Vol. 13, 1 (1992), 35-39.

[9] He LePing, GaO MingZhe, ZHOU Yu, On New Extensions of Hilbert's Integral Inequality, International Journal of Mathematics and Mathematical Science, Volume 2008, Article ID 297508, 8 pages, 2008.

[10] Yang Bicheng, A New Hilbert Type Integral Inequality and Its Generalization, J. Jilin Univ. (Sci. Ed.), Vol. 43, 5 (2005), 580-584.

[11] M. KRnić, J. PEČARIĆ, General Hilbert's and Hardy's inequalities, Math. Inequal. Appl. 8 (2005), $29-52$.

[12] M. Krnić, G. Mingzhe, J. PeČArić, G. Xuemei, On the best constant in Hilbert's inequality, Math. Inequal. Appl. 8 (2005), 317-329.

[13] I. Perić, P. Vuković, Hardy-Hilbert's inequalities with a general homogeneous kernel, Math. Inequal. Appl. 12 (2009), 525-536.

[14] Wang Lianxiang, Fang Dexhi, Mathematical Handbook, People's Education Press, Beijing, 1979. 